\title{
Have the heavy neutrinos gone?
}

\section{The pursuit of particle physics without the use of accelerating machines is always an intriguing challenge. But the simplicity of measurements may be offset by complications of interpretation.}

THE search for the heavy neutrino continues, but the chances of success seem steadily to diminish. That is the curious psychological effect of a demonstration, just published, of the pitfalls ahead of those who look for evidence of heavy neutrinos (where "heavy" merely means "greater than zero") in the electron spectrum associated with beta-decay.

The argument, due to J. Lindhard and P. G. Hansen from the University of Aarhus in Denmark (Phys. Rev. Lett. 57, $965 ; 1986)$ is interesting not so much because it is decisive, but because of its simplicity and clarity. Its bearing on the question whether meutrinos can have even a modicum of mass is only indirect.

The idea that neutrinos may have mass is now some 15 years old. The successful unification of the weak and the electromagnetic force-fields implies that there should be as many kinds of neutrinos as there are different kinds of electron-like particles, which for the time being means three (the tauon and muon with the ordinary electron). The notion that some neutrinos may have mass arises from attempts to unify strong and weak nuclear forces.

Cosmologists do not bother to conceal their delight at the prospect that a nearly universally distributed particle might carry enough mass to help close the Universe gravitationally. Everybody seems to agree that, if there are indeed three kinds of neutrinos with very similar properties, those actually observed will be mixtures (in the quantum sense of being linear combinations of fundamental states) of the three basic neutrinos. Specifically the neutrinos (strictly, anti-neutrinos) emitted in partnership with electrons during beta-decay will on the average carry a fraction of any mass there may be.

This was the inspiration of an accurate measurement 10 years ago by Soviet physicists of the energy-spectrum of electrons emitted in the beta-decay of tritium. The point is merely that if the associated neutrinos have even the smallest amount of mass, the requirement that energy and momentum should be conserved in the decay will distort the energy spectrum that would otherwise be expected.

As neutrinos as such cannot be observed directly because they hardly interact with matter, the predicted distortion of the electron energy-spectrum may offer the best hope, for the time being, of measuring their properties. A quick calculation will show that there will be a ten-. dency for an excess of electrons at low energy.

Tritium is an obvious place to start, if only because the transition energy is relatively small and because the effect on the decay process of the atomic electron shell can be calculated easily, given the simplicity of the structure. But the same search for distorted energy spectra has been carried out with the isotopes ${ }^{64} \mathrm{Cu},{ }^{35} \mathrm{~S}$ and no doubt others.

Most experiments have yielded only inconclusive evidence. Datar, V.M. et al. (Nature 318, 547; 1985), for example, working with ${ }^{35} \mathrm{~S}$, found that the admixture of a single supposedly massive neutrino corresponding to, say, a muon with the supposedly zero-mass electron neutrino would be, at most, a mere 1 per cent.

The outstanding result in the field remains that of J.J.Simpson, from the University of Guelph in Ontario, who has studied tritium decay by a neat technique in which tritium atoms are embedded in a silicon detector that functions as a means of measuring the total loss of energy by ionization within the silicon, for which purpose the semiconductor is doped with lithium (Phys. Rev. Lett. 54, 1891; 1985).

Simpson's conclusion, going against the grain of earlier evidence except that from the Soviet Union, is that the energy spectrum of the decay electrons does indeed differ from that expected on the simplest principles, and in such a way as to suggest that the massless neutrino is mixed to the extent of 3 per cent with a heavier neutrino whose mass is the equivalent of approximately $17.2 \mathrm{keV}$. Ungratefully, even cosmologists are not enthusiastic: this neutrino is embarrassingly massive.

Lindhard and Hansen make one elementary point about Simpson's experiment, that it differs from most others of this kind in that the decaying tritium nuclei embedded in silicon are mostly stripped bare of their single electron. It follows that the decay energy is not the conventional $18.6 \mathrm{keV}$, the difference of energy between an atom of tritium (with one electron) and an atom of its decay product, ${ }^{3} \mathrm{He}$, which has two electrons.

Most simply, what this remark implies is that the energy available in the decay of a stripped tritium nucleus is less than that which might be wrung from the decay of an intact atom by the difference between the absolute values of the two groundstate electron energies, both negative (of which that of helium is larger to the tune of more than $65 \mathrm{eV}$ ). But to say that the energy available will be different for the decay of a stripped and for an intact atom does not imply that all the difference will be conferred on the decay electrons, although the magnitude of the effect is such as to suggest that Simpson's small differences could easily be swamped.

What Lindhard and Hansen have done is to make qualitative calculations of how the difference of available energy between stripped and intact atoms will be reflected in the energy spectrum of the electrons. The most obvious influence is that of the atomic electron in an intact tritium atom, which will have the effect of increasing the outward energy of the electron. Other effects of the atom on the departing electron are more subtle.

The fact that the sudden conversion, in beta-decay, of a tritium nucleus to a helium nucleus leaves the lone electron in an excited state implies that some part of the $18.6 \mathrm{keV}$ difference of energy between the two ground states is released not to the electron or neutrino, but in de-excitation. Similarly with the deionization that must follow that adjustment. Note that the term "sudden" is not merely descriptive but is a technical term in quantum mechanics.

The calculation of the quantities involved is unavoidably messy; that for the screening correction was first done by M.E. Rose in the 1930s. Lindhard and Hansen add a nice twist by using a function to represent the screening potential of an electron shell that includes an exponential term yet nevertheless allows for exactly soluble wave functions.

The upshot is the conclusion that the energy carried off by electrons will be decreased, on the average, by some $34 \mathrm{eV}$, or that the expected electron energy spectrum calculated from the full value of $18.6 \mathrm{keV}$ will be shifted bodily towards the origin of energy by that amount. This, the authors claim, is enough to account for two-thirds of the discrepancy claimed by Simpson. Chemical interactions, weighing in on the same side as de-excitation and deionization, could account for the rest.

There is no logical reason why this should strengthen beliefs that massive neutrinos do not exist. The theories are explicit on the point, while there are many other types of experiments in which the issue may be tested. But there is a sense in which negative trials of a hypothesis count positively against it: many people are prejudiced against failure.
John Maddox 